\title{
RNA Expression Profiling of Lymphoepithelioma-Like Carcinoma of the Bladder Reveals a Basal-Like Molecular Subtype
}

Ujjawal Manocha, ${ }^{*}$ Jordan Kardos, ${ }^{*}$ Sara Selitsky, ${ }^{*}$ Mi Zhou, ${ }^{\star}$ Steven M. Johnson, ${ }^{\dagger}$ Cori Breslauer, ${ }^{\dagger}$ Jonathan I. Epstein, ${ }^{\dagger}$ William Y. Kim, ${ }^{*} \AA$ and Sara E. Wobker ${ }^{\star \dagger}$

From the UNC Lineberger Comprehensive Cancer Center, $*$ and the Departments of Pathology and Laboratory Medicine, ${ }^{\dagger}$ Medicine, ${ }^{\S}$ and Genetics, University of North Carolina at Chapel Hill, Chapel Hill, North Carolina; and the Departments of Pathology, Urology and Oncology, ${ }^{\ddagger}$ Johns Hopkins Hospitals, Baltimore, Maryland

Accepted for publication September 5, 2019.

Address correspondence to Sara E. Wobker, M.D., M.P.H., 303 Brinkhous-Bullitt Bldg., CB\#7525, Chapel Hill, NC 27599. E-mail: sara wobker@med.unc.edu.

\begin{abstract}
Lymphoepithelioma-like carcinoma of the bladder (LELC-B) is a rare subtype of urothelial carcinoma consisting of undifferentiated epithelial cells within a dense inflammatory cell infiltrate. We set out to molecularly characterize LELC-B through RNA expression profiling as well as immunohistochemistry (IHC) to understand its underlying biology. Sixteen cases of LELC-B were identified at Johns Hopkins University. RNA sequencing was performed on 14 cases. IHC staining for programmed cell death ligand 1 (PD-L1) and mismatch repair proteins MutL homolog 1 (MLH1), MutS homolog 2 (MSH2), MSH6, and PMS1 homolog, mismatch repair system component 2 (PMS2) was performed. Transcriptomic profiling of LELC-B showed that they are enriched in a basal-like phenotype, with 12 of 14 LELC-B cases correlating to the basal centroid of the bladder cancer analysis of subtypes by gene expression 47 (BASE47) predictive analysis of microarrays (PAM) classifier. Gene signature analysis confirmed the lymphocyte infiltration profile consistent with the histomorphology. LELC-B lacked features to explain the robust lymphocytic infiltrate, such as loss of mismatch repair protein expression or expression of Epstein-Barr virus transcripts. Nonetheless, PD-L1 IHC was positive in $93 \%$ of LELC cases. Our study demonstrates that LELC-B tumors are enriched in a basal-like molecular subtype and share a high level of immune infiltration and PD-L1 expression, similar to basal tumors. The basal-like phenotype is consistent with the known sensitivity of LELC-B to chemotherapy and suggests that immune checkpoint therapy should be explored in this rare disease. (Am J Pathol 2020, 190: 134-144; https://doi.org/10.1016/ j.ajpath.2019.09.007)
\end{abstract}

Lymphoepithelioma-like carcinoma of the bladder (LELC-B) is a rare variant of urothelial carcinoma composed of sheets or nests of undifferentiated cells with ill-defined cytoplasmic borders and large pleomorphic nuclei with prominent nucleoli. The tumor cells are present within a dense infiltrate of predominantly lymphocytes, plasma cells, and other inflammatory cells. ${ }^{1,2}$ This entity was first described in 1991, and it represents just $0.3 \%$ to $1.4 \%$ of all bladder carcinomas. ${ }^{3}$ The histologic appearance of LELC-B closely resembles lymphoepithelioma of the nasopharynx, which is defined by both its histologic appearance and presence of Epstein-Barr virus (EBV) positivity. In bladder, LELC was first shown to be EBV negative via absence of EBV staining by in situ hybridization to EBV-encoded RNA (EBER1). ${ }^{4}$ It remains uncertain what factor is promoting the immune infiltration of these EBVnegative tumors.

Pure or predominant LELC-B (>50\% LELC-B features) ${ }^{1}$ has shown better overall survival compared with tumors displaying $<50 \%$ LELC, but the mechanism for this finding is not yet understood. On the basis of a systematic review of LELC-B in the literature, Yoshino et $\mathrm{al}^{5}$ reported an overall

\footnotetext{
Supported by the University Cancer Research Fund (W.Y.K and S.E.W. and in part by National Cancer Institute grant 5P30CA016086-42, NIH grant U54-CA156733, National Institute of Environmental Health Sciences grant 5 P30 ES010126-17, the University Cancer Research Fund, and North Carolina Biotechnology Center grant 2015-IDG-1007.

Disclosures: None declared.
} 
cause-specific survival rate of $83 \%$ for LELC-B, with $97 \%$ and $95 \%$ survival in pure and predominant patterns, respectively, compared with $15 \%$ survival in those patients with only focal LELC-B pattern.

Another histologic analog of LELC-B is the medullary phenotype of colorectal carcinoma. These colon tumors show minimal to no glandular growth and are composed of sheets and nests of epithelial cells that have large, pleomorphic nuclei and prominent nucleoli, embedded within a dense lymphocytic infiltrate. ${ }^{6}$ Interestingly, the medullary phenotype of colorectal carcinoma is strongly associated with a high degree of microsatellite instability, showing loss of DNA mismatch repair (MMR) proteins. ${ }^{7}$ Despite this loss, these tumors are often localized and have a better prognosis than other colorectal cancer subtypes. ${ }^{8}$ In addition, tumors with a high degree of microsatellite instability are often found to express programmed cell death ligand 1 (PD-L1), which may be a direct result of tumor infiltrating lymphocytes. ${ }^{9}$

The expression of PD-L1 on tumor cells interacts with its ligand to inhibit T-cell activation. Recently, PD-L1 expression has been the focus of studies evaluating its use as a marker of response on tumor cells and tumor-infiltrating lymphocytes. Consequently, it is believed that the overexpression of PD-L1 leads to worse outcomes in numerous tumor types, including urothelial carcinoma. ${ }^{10}$ PD-L1 expression in tumor cells has been associated with a worse disease-specific and overall survival. ${ }^{11}$

High-grade, muscle invasive bladder cancer demonstrates clinically relevant molecular subtypes based on RNA expression. ${ }^{12-16}$ Although the precise number of subtypes has yet to be determined, there is broad agreement that overarching basal and luminal subtypes exist. ${ }^{17}$ The basal, luminal, luminal-infiltrated, and claudin-low molecular subtypes of urothelial carcinoma have emerged as important prognostic markers and may predict response to neoadjuvant chemotherapy. ${ }^{13,18}$ Overall survival appears better in luminal tumors, whereas better response to neoadjuvant chemotherapy in basal tumors leads to improvements in survival over surgery alone. ${ }^{18}$ Conversely, claudin-low tumors appear to have a worse prognosis compared with the other subtypes, regardless of neoadjuvant therapy. ${ }^{18}$

The molecular and immunohistochemical (IHC) profiles of urothelial cancers with variant histology are beginning to yield important results. Micropapillary urothelial carcinoma is a particularly aggressive variant and has been assigned a luminal subtype, has unique miRNA expression patterns, and demonstrates high rates of ERBB2 (HER2) amplification and activating mutation. ${ }^{19,20}$ Another aggressive variant, plasmacytoid urothelial carcinoma, shows near universal loss-offunction mutations in $\mathrm{CDH} 1$, which codes for the cell adhesion protein E-cadherin. ${ }^{21}$ This loss is thought to explain the tumor's tendency for aggressive spread beyond fascial planes. Partly because of the rarity of LELC-B, molecular characterization of LELC-B has not been explored. There are potential treatment implications to understanding the molecular subtypes of these rare variants, given that large-scale clinical trials are not feasible in this population.

Our aim was to profile LELC-B via immunohistochemistry and RNA sequencing. We investigated the immunohistochemical expression of PD-L1 and MMR proteins MLH1, MSH2, MSH6, and PMS2, as well as use PAM classifiers to classify LELC-B into the BASE47 and University of North Carolina at Chapel Hill (Chapel Hill, NC) subtypes of bladder cancer. We hypothesized that LELC-B shows enrichment in markers of the basal molecular subtype of bladder cancer because of the sensitivity to chemotherapy seen in LELC-B. Immune gene signature analyses were done to address the high immune infiltration in LELC-B.

\section{Materials and Methods}

\section{LELC-B Case Selection}

The study was performed with approval of the Institutional Review Boards at both University of North Carolina at Chapel Hill and Johns Hopkins (Baltimore, MD). Cases were identified by a search of the Johns Hopkins Pathology Lab Information System for cases containing the terms lymphoepithelioma-like carcinoma and bladder from January 1, 2000, to July 25, 2016. Inclusion criteria included patients with a surgical resection specimen from the bladder or transurethral resection of bladder tumor accessioned at Johns Hopkins, with a final diagnosis of LELC-B. Cases with only focal LELC-B were excluded from the study. LELC-B tumors were classified as pure or predominant using the cutoff of $>50 \%$ for predominant pattern LELC-B and 100\% LELC-B for pure pattern. ${ }^{1}$ Sixteen cases of pure $(n=12)$ or predominant $(n=4)$ LELC-B were identified in the archives (Table 1). Deidentified, unstained tissue sections were used for IHC and nucleic acid extraction.

\section{Immunohistochemistry}

Initial hematoxylin and eosin-stained slides (Figure 4A) were reviewed for tumor adequacy and stained with PD-L1 IHC (Cell Signaling, Boston, MA; E1L3N) and MMR proteins (MLH1, MSH2, MSH6, and PMS2; Cell Marque, Rocklin CA) in a single batch. Additional immunohistochemical stains [cytokeratin 5/6 (CK5/6), CK14, forkhead box A 1 (FOXA1), erythroid transcription factor 3 (GATA3), and uroplakins 2 (UPK2)] were performed by restaining the prior slides used for MMR and one hematoxylin and eosin-stained slide. The IHC staining for these five stains was interpreted independently by two pathologists (S.M.J. and S.E.W.) and any cases not reaching consensus were reviewed together to resolve discrepancies. CK5/6, CK14, FOXA1, GATA3, and UPK2 were given both a stain intensity and percentage of tumor cells staining positively. To develop the modified Allred score, these stains were given a score of 0 to 6 for percentage-positive 
tumor cells $(0$ indicates $0 \% ; 1,1 \%$ to $4 \% ; 2,5 \%$ to $20 \%$; $3,21 \%$ to $40 \%$; $4,41 \%$ to $75 \% ; 5,76 \%$ to $99 \%$; and $6,100 \%)$ and 0 to 3 for stain intensity ( 0 indicates none; 1 , weak; 2 , moderate; and 3, strong). Scores were added for a maximum of 9 . The $\mathrm{H}$-score was calculated by multiplying the stain intensity by the continuous percentagepositive tumor cells (possible range, 0 to 300$)^{22}$ (Supplemental Table S1). For MMR IHC, cases were designated as retained expression (any tumor nuclei staining positive at any intensity) or lost expression (negative in all tumor nuclei). For PD-L1, cases were interpreted using the tumor proportion score and combined positive score (CPS) method. CPS was developed for solid tumors stained with the PD-L1 22C3 clone as a way to capture both tumor cell and immune cell staining in one number, calculated as the number of PD-L1-positive cells (tumor cells, lymphocytes, and macrophages) divided by the total number of viable tumor cells, then multiplied by $100 .^{23}$ Although the CPS system is not formally validated for PD-L1 clones other than $22 \mathrm{C} 3$, it was used to express nontumor cell expression of PD-L1 in these cases.

\section{RNA Extraction and Data Set Processing}

Sixteen cases were identified with formalin-fixed, paraffinembedded tissue available for study. Fourteen cases had sufficient RNA yield after extraction. RNA was extracted with the Roche High Pure RNA Paraffin kit protocol (Roche Diagnostics, Indianapolis, IN). Fourteen samples generated adequate yield for the library preparation with the Illumina TruSeq RNA Access Library Prep Kit (Illumina, San Diego, CA) and were sequenced on an Illumina NextSeq500. FASTQ files from all cases in The Cancer Genome Atlas (TCGA) bladder cancer (TCGA BLCA) data set were obtained from database of Genotypes and Phenotypes (dbGap;

Table 1 Clinical Characteristics of LELC-B Tumor Samples

\begin{tabular}{llll}
\hline Case ID & Stage & Histology & Specimen \\
\hline SW1 & pT2 & Pure & TURBT \\
SW3 & pT2b & Pure & Cystectomy \\
SW5 & pT2 & Pure & TURBT \\
SW6 & At least pT1 ${ }^{*}$ & Pure & TURBT \\
SW7 & pT2 & Pure & TURBT \\
SW8 & pT2 & Pure & Ureterectomy \\
SW9 & pT2a & Pure & Cystectomy \\
SW10 & pT2 & Pure & TURBT \\
SW11 & pT2 & Pure & TURBT \\
SW12 & pT2 & Predominant & TURBT \\
SW13 & pT2b & Pure & Cystectomy \\
SW14 & At least pT1 ${ }^{*}$ & Predominant & TURBT \\
SW15 & At least pT1 & Pure & TURBT \\
SW16 & At least pT1 ${ }^{*}$ & Pure & TURBT \\
SW17A & pT2 & Predominant & TURBT \\
SW17B & pT2 & Predominant & TURBT \\
\hline
\end{tabular}

${ }^{*}$ No muscularis propria present for evaluation in TURBT specimen.

ID, identification; LELC-B, lymphoepithelioma-like carcinoma of the bladder; TURBT, transurethral resection of bladder tumor. https://www.ncbi.nlm.nih.gov/gap). TCGA BLCA along with 14 LELC-B samples were analyzed in $\mathrm{R}$ version 3.5.2 ${ }^{24}$ (Supplemental Figure S1A).

Normal cases were excluded, and subtype calls were acquired from TCGA group. ${ }^{15}$ Counts aligned to Human Genome Release 29 (GRCh38.p12) via STAR (2.5.3a) and quantified via Salmon (0.8.2). ${ }^{25-27}$ Gene expression data for LELC-B $(n=14)$ and TCGA $(n=408)$ were merged via tximport (1.8.0) and biomaRt (2.36.1). ${ }^{28,29}$ The data were filtered for genes that had expression in at least $80 \%$ of the samples $(n=422)$. The raw expression data were normalized to account for sequencing depth differences via size factor normalization and were $\log$ transformed via the DESeq2 package (1.20.0). ${ }^{30}$ Resulting data were adjusted for batch effects via Surrogate Variable Analysis (3.28.0) and Q3 normalized (Supplemental Figure S1B). ${ }^{31}$ The merged data set contained gene expression data from LELC-B $(n=14)$ and TCGA BLCA $(n=408)$ aligned to the hg38 genome. Principal component analysis was conducted to check for outliers (Supplemental Figure S2). All data were visualized via the use of ComplexHeatmap (1.20.0) and ggplot2 (3.1.0) vignettes. $^{32,33}$

\section{Molecular Characterization}

The merged LELC-B-TCGA BLCA data set $(n=422)$ was classified into basal, luminal, or claudin-low subtypes via the BASE47 and the BCL40 PAM Classifier (Supplemental Table S2). ${ }^{14,34,35}$ The Fisher exact test was used to determine significant associations for the BASE47 classifier. Expression data were Z-normalized, and the Pearson distance was used for hierarchical clustering. ${ }^{32}$ An unsupervised clustering heat map of the bladder cancer classification markers was generated. ${ }^{15}$ Gene signatures corresponding to an immune infiltrated tumor subtype via $\mathrm{B}$ cells and $\mathrm{T}$ cells were investigated in LELC-B. ${ }^{36}$ Significance was tested by Wilcoxon-Mann-Whitney rank sum method as it does not assume a parametric distribution. Further characterization of LELC-B was performed via a comparison to Urothelial Carcinoma via Gene Set Enrichment Analysis and the MSigDB Hallmark Gene Set Collection ${ }^{37,38}$ (Supplemental Figure S3B and Supplemental Table S3). Just for the Gene Set Enrichment Analysis, three cases were removed from TCGA BLCA data set (one case each for adenomas/adenocarcinomas, epithelial neoplasm not otherwise specified, and squamous cell neoplasm) to yield a data set of $n=405$ urothelial carcinoma samples.

\section{Detection of Epstein-Barr Virus}

Epstein-Barr virus (human herpesvirus 4/EBV) was detected using VirDetect. ${ }^{39}$ VirDetect aligns reads against a curated and masked viral genome database composed of 1893 vertebrate virus reference genomes from GenBank via STAR RNAsequencing aligner. ${ }^{26}$ All reads in the sample FASTQ file that did not align to the hg38 genome were aligned to the viral 
genome collection. Our LELC samples were assessed for wildtype EBV (accession identifier: NC_007605.1) and EBV type 2 (accession identifier: NC_009334.1) along with cell lines with validated $\mathrm{EBV}$ presence. ${ }^{40}$ The alignments of the tumor sequence to the viral genomes were verified with human endogenous retrovirus as a positive control (accession identifier: NC_022518.1). All sequences used can be referenced via the accession numbers through the National Center for Biotechnology Information website (https://www.ncbi.nlm. nih.gov/nuccore).

\section{Results}

\section{LELC-B Is Enriched in Markers Corresponding to Basal-} Like Bladder Cancer

High-quality RNA was obtained from 14 pure or predominant LELC-B tumors, most of which initially presented with muscle invasion (Table 1). Examination of the LELC-B tumors by BASE47 showed that most of LELC-B has a significant correlation toward the basal centroid, with 12 LELCB tumors classified as basal-like and 2 LELC-B tumors being classified as luminal-like bladder cancer (Fisher exact $P=0.0117$ ) (Figure 1A and Supplemental Table S2). Eight of 10 cases of pure LELC-B and four of four predominant cases correlated to the basal centroid. The PAM classification results were confirmed by unsupervised clustering on the genes that highlight the subtypes within muscle invasive bladder cancer (Figure 2A). ${ }^{15}$

A subset of basal tumors have a claudin-low phenotype akin to the breast cancer claudin-low subtype. ${ }^{34,41}$ It was determined whether any of the basal-like LELC-B tumors showed an enrichment in markers of the claudin-low subtype of bladder cancer using the previously published BCL40 PAM Classifier. ${ }^{34}$ None of the LELC-B basal tumors were characterized as the claudin-low subtype (Figure 1B). Therefore, although LELC-B tumors exhibit a basal phenotype, they are not claudin-low. The hallmark genes that are representative of bladder cancer subtypes, such as GATA3 and FOXA1, confirm that LELC-B is significantly different from the luminal and claudin-low profile (Figure 2B) and most in keeping with a basal-like subtype.

\section{LELC-B Phenocopies Basal Bladder Cancer via Immune Gene Signatures}

The expression of a panel of immune gene signatures that have previously been shown to be representative of differing components of the immune system was examined next. ${ }^{36,42}$ Representative Z-scores of immune gene signatures in a heat map supervised by University of North Carolina at Chapel Hill subtype and rank ordered within each subtype were used (Supplemental Figure S3A). ${ }^{36,42,43}$ Although the LELC-B tumors overall appeared to have heterogeneous expression levels of these immune gene signatures, in
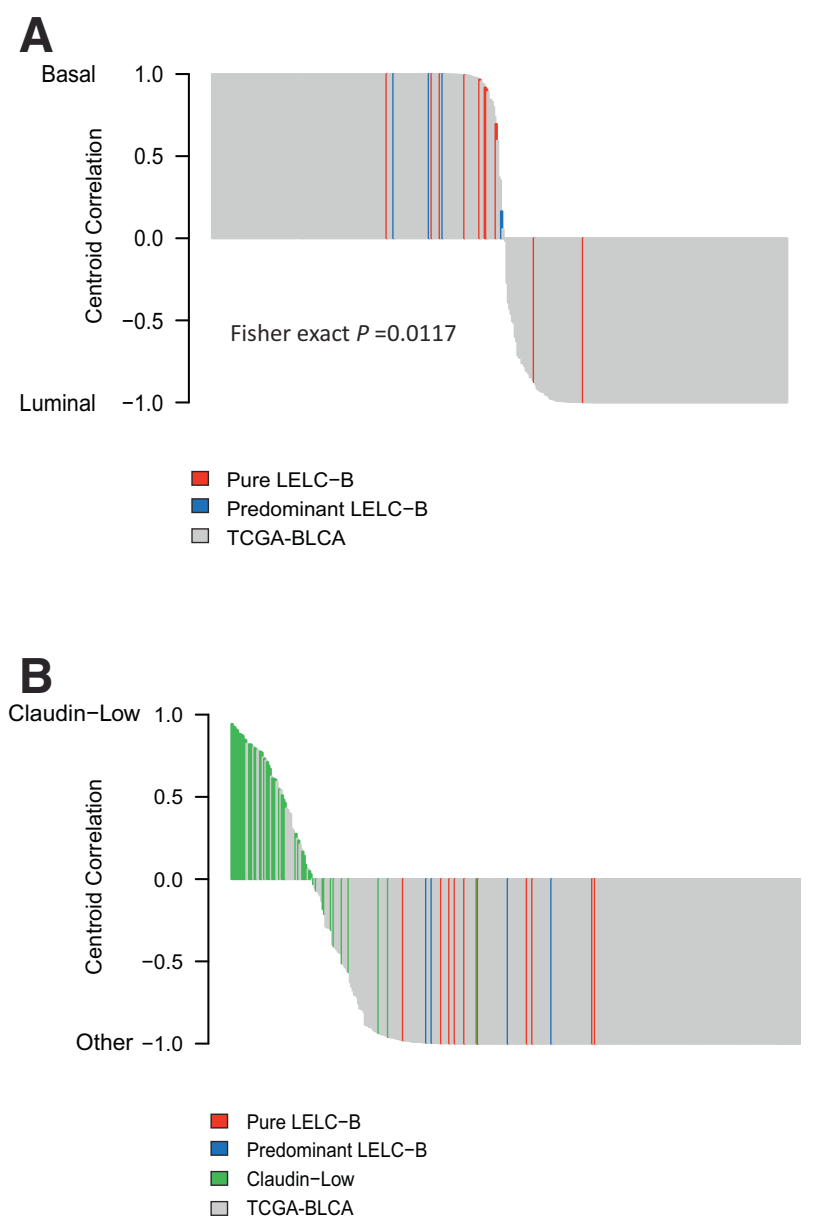

Figure 1 A: Waterfall plot for the BASE47 PAM classification of lymphoepithelioma-like carcinoma of the bladder (LELC-B) compared with The Cancer Genome Atlas bladder cancer (TCGA BLCA) data set consisting of muscle-invasive tumors. ${ }^{14}$ LELC-B tumors were significantly correlated to the basal centroid (Fisher exact test; $P=0.0117$ ). B: Waterfall plot for BCL40 PAM classifier. ${ }^{34}$ LELC-B is not associated with the claudin-low tumor subtype. $n=408$ (A).

general, LELC-B tumors showed a profile of active immune infiltration. To verify this, the expression of T-cell, B-cell, and an immunosuppression signature was specifically examined. In all three signatures, LELC-B tumors showed gene signature levels similar to basal urothelial carcinoma tumors, yet significantly higher and lower than luminal and claudin-low tumors, respectively (Figure 3 ). These findings are consistent with the notion that LELC-B tumors have RNA expression patterns similar to basal-like bladder tumors.

\section{LELC-B Has High Tumor Cell PD-L1 Expression}

To further classify the immune landscape of LELC-B, immunohistochemistry was performed for PD-L1 (Figure 4B). In 15 of 16 total cases (one case was excluded because of inadequate tissue), 14 (93\%) were determined to have positive tumor cell staining for PD-L1 (nine demonstrating high expression and five demonstrating low 
A

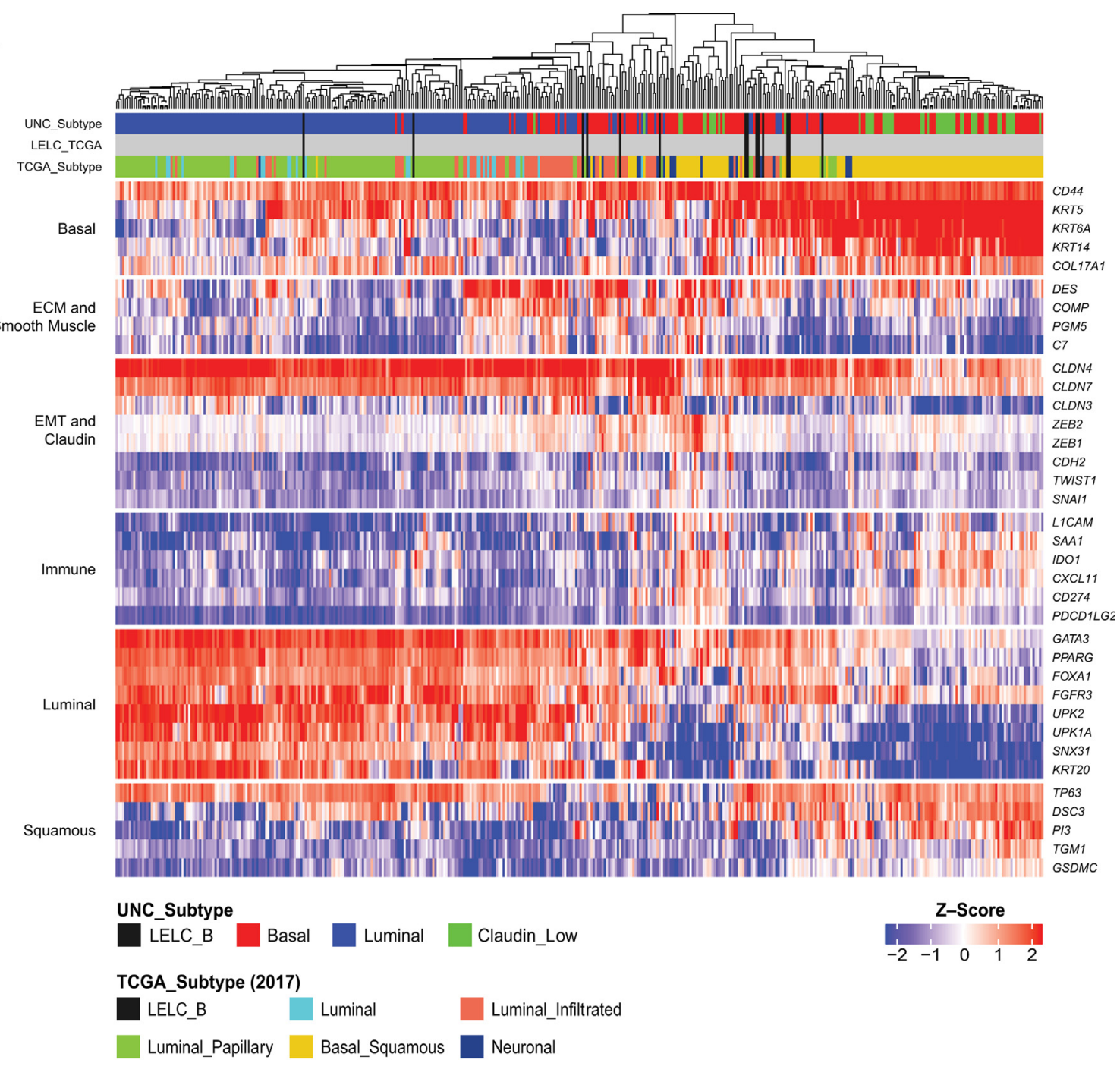

$\mathbf{B}$
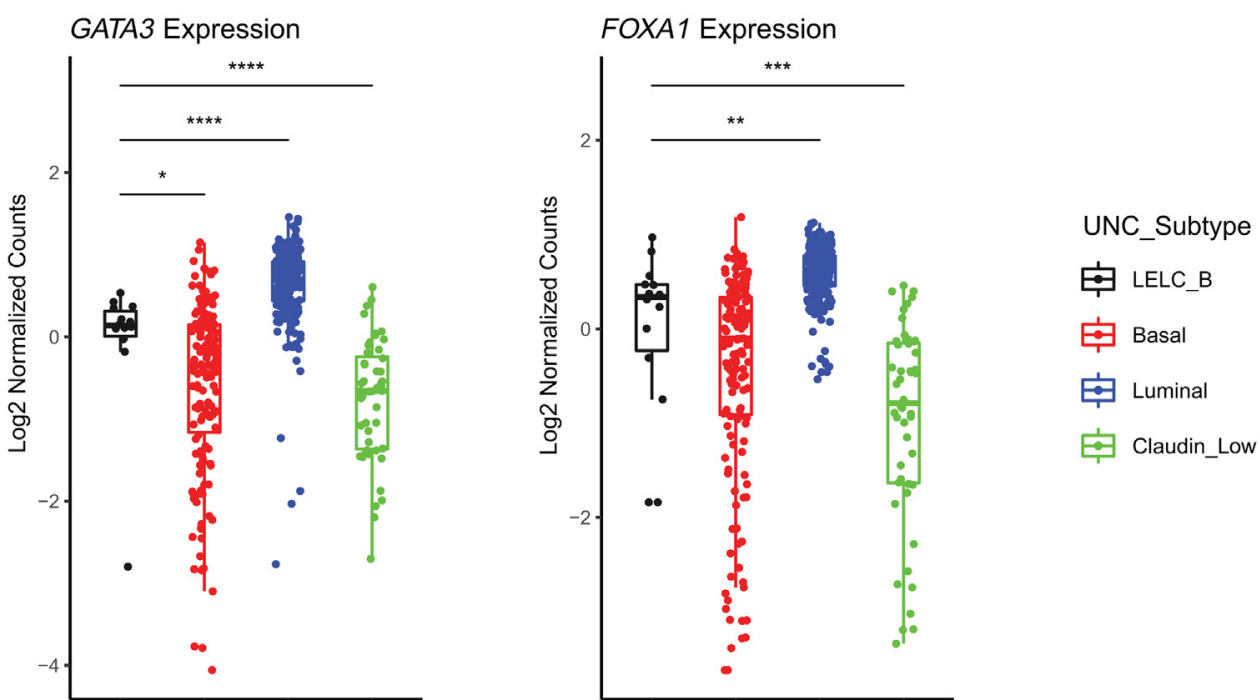

Figure 2 A: Unsupervised clustering of the merged data set on genes characteristic of muscle-invasive bladder cancer. ${ }^{15}$ Pearson correlation and average linkage were used on Z scores for the 47 genes. B: Supervised clustering on normalized expression counts of FOXA1 and GATA3 within the University of North Carolina at Chapel Hill (UNC) subtype scheme. ${ }^{*} P<0.05,{ }^{* *} P<0.01,{ }^{* * *} P<0.001$, and ${ }^{* * *} P<0.0001$ (Wilcoxon-Mann-Whitney rank sum test). ECM, extracellular matrix; EMT, epithelial-mesenchymal transition; LELC, lymphoepithelioma-like carcinoma; LELC-B, LELC of the bladder; TCGA, The Cancer Genome Atlas. 

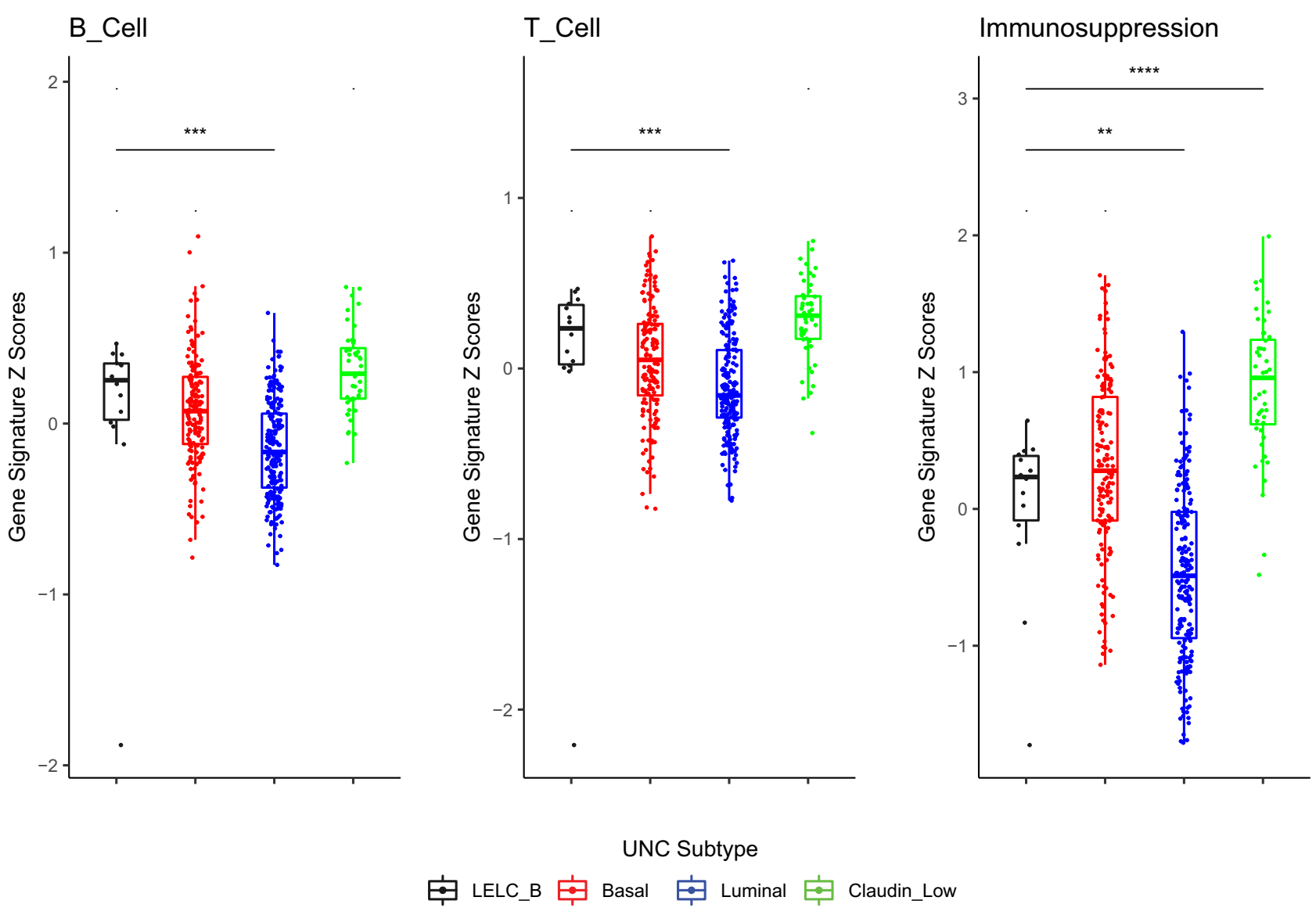

Figure 3 Box plots for gene signature Z scores corresponding to B-cell activation, T-cell activation, and immunosuppression. ${ }^{34,36}$ Lymphoepithelioma-like carcinoma of the bladder (LELC-B) is significantly different from luminal and claudin-low subtypes, but not basal bladder cancer. ${ }^{* *} P<0.01,{ }^{* * *} P<0.001$, and $* * * P<0.0001$ (Wilcoxon-Mann-Whitney rank sum test).

expression) (Table 2). The five cases with low expression were all pure LELC-B, whereas the nine cases with high expression included seven pure and two predominant types. The single PD-L1-negative case was a predominant LELC-B. There was no significant association between pure or predominant LELC-B cases and PD-L1 IHC status via Fisher exact test $(P=0.505)$. According to the CPS interpretation, all 14 LELC-B tumors that expressed PD-L1 scored $\geq 10$, which is positive overall. The IHC images demonstrate PD-L1 expression in LELC-B tumor cells, with lack of expression in the infiltrating lymphocytes (Figure 4B).

\section{LELC-B Has Intact MMR Protein Expression}

All 16 cases of LELC-B uniformly retained MMR protein expression, the only exception being the loss of MSH6 in a single case of a pure pattern LELC-B tumor. Figure 4, C-F, depicts photomicrographs demonstrating representative cases for each IHC marker: MLH1, MSH2, PMS2, and MSH6. There were no significant associations between MMR intact status and PD-L1 expression. Therefore, although MMR deficiency may explain the robust immune infiltrate in medullary colorectal carcinoma, LELC-B does not appear to be MMR deficient, at least when assessed by immunohistochemistry.

\section{LELC-B Does Not Associate with Epstein-Barr Viral Co- Infection}

A final potential explanation for the robust immune infiltrate associated with LELC-B is the expression of a pathogenic virus (ie, EBV). Although previous studies have shown that LELC-B tumors do not express EBV by EBER staining, this question was asked in an orthogonal manner by querying the RNA-sequencing data for expression of the EBV genome. ${ }^{4}$ To examine EBV presence, reads were aligned to a masked and curated database of vertebrate genomes from GenBank (Materials and Methods). Alignment to human herpesvirus 4 genome (EBV) sequence showed a lack of EBV transcripts in LELC-B sequencing reads (Table 3). As expected, counts aligning to human endogenous retrovirus K113 were present in all samples tested, validating its use as a positive control. RNA expression from known EBV expressing cell lines was included to confirm that this method detects EBV 

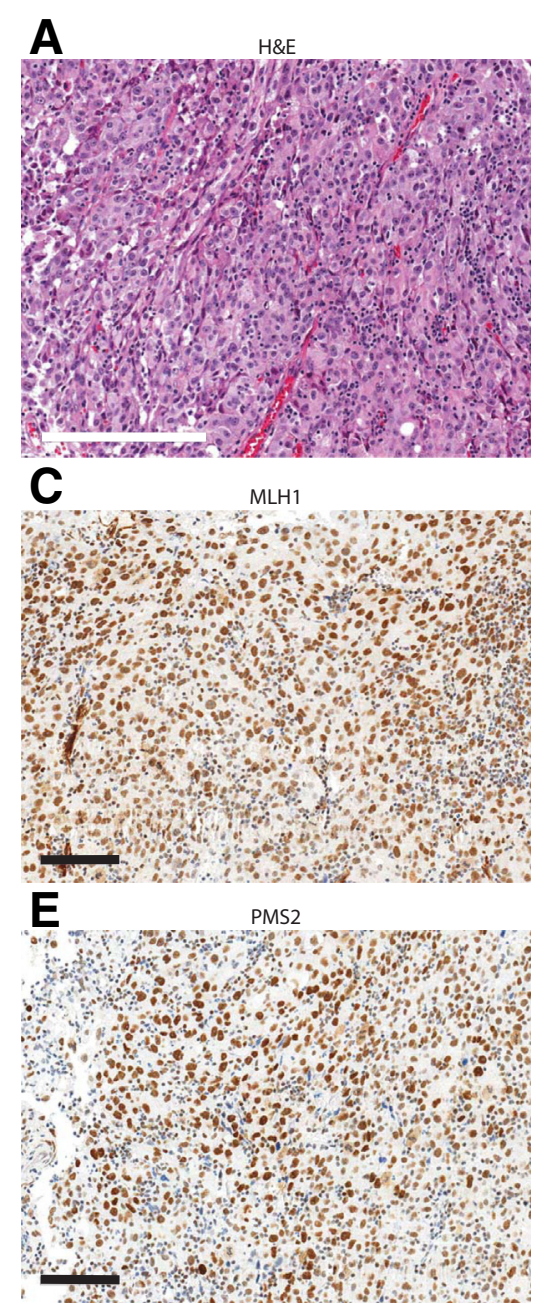
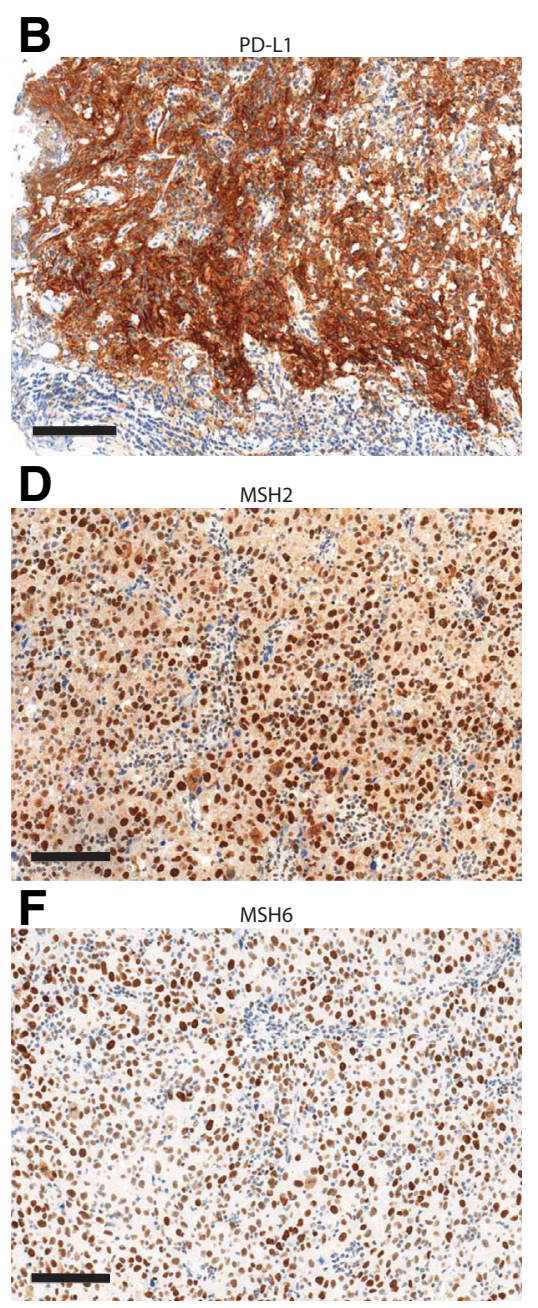

Figure 4 A: Hematoxylin and eosin (H\&E)-stained section of lymphoepithelioma-like carcinoma of the bladder, showing large epithelioid cells in a mixed lymphoid background. B: Programmed cell death ligand 1 (PD-L1) immunohistochemical (IHC) staining demonstrating high positive staining. C-F: MLH1, MSH2, PMS2, and MSH6 IHC all demonstrating retained staining. Scale bars: $200 \mu \mathrm{m}(\mathbf{A}) ; 100 \mu \mathrm{m}(\mathbf{B}-\mathbf{F})$. transcripts (Table 3). These results are consistent with prior studies that used in situ hybridization to EBERI to detect EBV presence and support in an orthogonal manner that LELC-B tumors do not harbor EBV.

\section{Discussion}

Histologic variants of urothelial carcinoma are relatively rare, with approximately $15 \%$ to $25 \%$ of bladder cancers containing morphologic patterns distinct from conventional urothelial carcinoma. ${ }^{4,45}$ The World Health Organization specified these variants in their 2016 monograph, and the consistent reporting of variant histology has been encouraged by all pathologists. ${ }^{46}$ An understanding of urothelial variants is critical for diagnostic, prognostic, and therapeutic reasons. ${ }^{47}$ Many of these variants have distinct natural histories, with micropapillary, plasmacytoid, and sarcomatoid variants associated with poorer outcomes. To our knowledge, this is the first study investigating the molecular characterization and immune profiling of LELC of the bladder.

Molecular characterization of high-grade bladder cancers, specifically intrinsic molecular basal and luminal phenotypes, is increasingly recognized as prognostic and predictive biomarkers. ${ }^{12-14}$ For example, multiple groups have demonstrated that the luminal subtype has a significantly better overall survival with or without neoadjuvant chemotherapy than the basal and claudin-low subtypes. ${ }^{18}$ In addition, one study has suggested that the basal subtype may be more responsive to platinum-based chemotherapy. ${ }^{18}$ Finally, because of differential immune infiltration, the subtypes might also be primed for immune checkpoint inhibitor response, although this has yet to be confirmed. On the basis of these observations and others, some groups have molecularly subtyped both variant and divergent histologic subtypes of bladder cancer in hopes of better understanding the underlying biology and potential implications for treatment choice.

Using the BASE47 gene set predictor, LELC-B cases show a basal-like phenotype. The basal subtype is so termed 
Table 2 Summary of LELC-B Immunohistochemistry Results

\begin{tabular}{ll}
\hline Characteristic & LELC-B cases \\
\hline$n$ & 16 \\
PD-L1 staining (IHC), $n$ (\%) & $9(60)$ \\
$\quad$ High & $5(33)$ \\
$\quad$ Low & $1(7)$ \\
$\quad$ No expression & \\
MMR deficiency (IHC), $n(\%)$ & $0(0)$ \\
$\quad$ MutL homolog 1 (MLH1) & $0(0)$ \\
MutS homolog 2 (MSH2) & $1(6)$ \\
MutS homolog 6 (MSH6) & $0(0)$ \\
PMS1 homolog 2 (PMS2) &
\end{tabular}

IHC, immunohistochemistry; LELC-B, lymphoepithelioma-like carcinoma of the bladder; MMR, mismatch repair; PD-L1, programmed cell death ligand 1.

because of its increased expression of basal keratin markers (KRT5, KRT6, and KRT14), leading to a more basaloid/stem cell phenotype. ${ }^{14}$ Although the basal subtype of bladder cancer confers a worse prognosis overall, it appears to show increased sensitivity to chemotherapy compared with nonbasal subtypes. ${ }^{18}$ LELC-B is historically thought to be exquisitely sensitive to chemotherapy and, although merely correlative, the enrichment of LELC-B in the basal subtype is at least consistent with this chemoresponsive phenotype.

Hematoxylin and eosin stains of LELC demonstrate high lymphocytic infiltrate, and previous work has shown that the lymphocytes are predominantly $\mathrm{CD} 8^{+}$and $\mathrm{CD} 4^{+} \mathrm{T}$ cells. ${ }^{2}$ In our studies, the immune gene signatures were also broadly up-regulated in LELC-B and had levels comparable to the basal-like urothelial carcinoma. Investigation of mechanisms underlying this robust lymphocytic infiltrate suggested no evidence for loss of MMR proteins or the presence of active EBV transcription. Unfortunately, tumor mutational burden and neoantigen load could not be assessed as DNA sequencing could not be performed on the LELC-B samples. Therefore, although these studies confirm the presence of an immune infiltrate within the tumor microenvironment, they do not elucidate the underlying drivers of this phenotype or define the relative clonality of the T cell. Nonetheless, it is tempting to speculate that the lymphocytic infiltrate may represent a robust, but exhausted, antitumor immune response that could be reinvigorated through immune checkpoint therapy.

Immune checkpoint inhibitors are being investigated as second-line therapy after platinum-based chemotherapy in metastatic bladder cancer. In the IMvigor 210 clinical trial, atezolizumab, an anti-PD-L1 antibody demonstrated a correlation between PD-L1 expression on tumor-infiltrating lymphocytes with longer overall survival. ${ }^{48}$ According to the PD-L1 IHC results, immune cells in LELC-B do not stain for PD-L1 (Figure 4B). However, the CheckMate275 trial showed that nivolumab, an antibody to programmed cell death protein 1 (PD-1), led to response regardless of high or low PD-L1 CPS scores in metastatic urothelial carcinoma. ${ }^{49}$ Similarly, pembrolizumab, a PD-1 antibody, showed clinical benefit but was not associated with high PD-L1 CPS. ${ }^{50}$ With immune checkpoint blockade becoming a prominent second-line therapy, further research is needed to investigate the dense tumor lymphocytic infiltrate seen histologically in LELC-B and any associations with better prognosis of pure and predominant LELC-B. ${ }^{5}$

Table 3 Viral Genome Quantification through STAR, including EBV (HHV-4) $)^{28}$

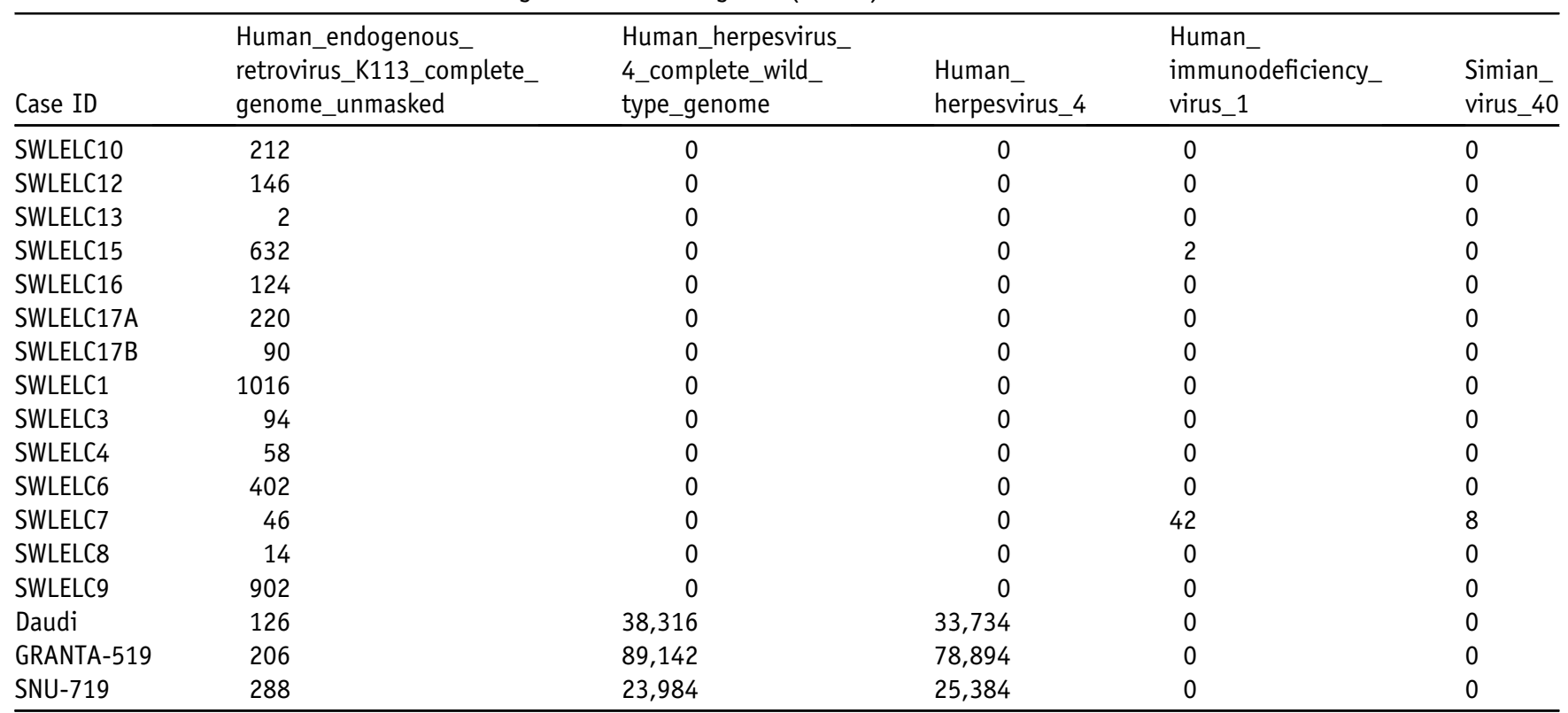

Lymphoepithelioma-like carcinoma of the bladder does not contain sequence aligning to EBV, which is concurrent with previous studies. ${ }^{2,4}$ RNA-sequencing data for cell lines validated to contain EBV were obtained from Klijn et al. ${ }^{40}$

EBV, Epstein-Barr virus; HHV-4, human herpesvirus 4; ID, identification. 
The limitations of our study are inherent in the use of formalin-fixed, paraffin-embedded archival tissue for RNA analysis. Biases may be present in data as RNA extracted from formalin-fixed, paraffin-embedded slides has lower yields and integrity than flash frozen tissue RNA. The data set processing removes any technical batch effects and minimizes bias. In any future studies, immune cell PD-L1 levels specifically should be investigated to determine whether LELC-B could be a candidate for immune checkpoint inhibitors. Further explanation for the robust immune infiltration should also be examined, such as the somatic mutational load or neoantigen burden. Additional investigation of a larger series of LELC-B cases with clinicopathologic outcomes should be attempted to confirm this study's findings and expand on the results.

In summary, the immune infiltration and immunosuppression profile in LELC is consistent with the low causespecific mortality rate; however, the immune gene signature expression was lower than expected. With Food and Drug Administration approval of immune checkpoint inhibitors for bladder cancer, the knowledge of the immune profile of the tumor is increasingly pertinent in treatment decision making. ${ }^{51}$ The robust lymphocytic infiltrate in LELC may be predictive as immune checkpoint blockade therapies gain Food and Drug Administration approval as second-line therapies for muscle invasive bladder cancers. ${ }^{52}$ Ongoing studies include RNA sequencing and further profiling of LELC-B tumors.

\section{Acknowledgments}

We thank the members of the Kim Laboratory for continued help and discussion throughout the project; Yongjuan Xia (University of North Carolina at Chapel Hill Translational Pathology Laboratory) for expert technical assistance; and the Department of Pathology at Johns Hopkins Hospitals for providing the formalin-fixed, paraffin-embedded tumor set.

\section{Author Contributions}

S.E.W. designed the study, performed literature search, collected data, and wrote the manuscript; U.M. generated and analyzed data, performed literature search, generated the figures, and wrote the manuscript; J.K. interpreted and analyzed the data; M.Z. analyzed the data; S.J. generated and analyzed data and reviewed the manuscript; C.B. generated and analyzed data; S.S. generated and analyzed data and reviewed the manuscript; W.Y.K. designed the study and reviewed the manuscript.

\section{Supplemental Data}

Supplemental material for this article can be found at http://doi.org/10.1016/j.ajpath.2019.09.007.

\section{References}

1. Amin MB, Ro JY, Lee KM, Ordóñez NG, Dinney CP, Gulley ML, Ayala AG: Lymphoepithelioma-like carcinoma of the urinary bladder. Am J Surg Pathol 1994, 18:466-473

2. Williamson SR, Zhang S, Lopez-Beltran A, Shah RB, Montironi R, Tan P-H, Wang M, Baldridge LA, MacLennan GT, Cheng L: Lymphoepithelioma-like carcinoma of the urinary bladder: clinicopathologic, immunohistochemical, and molecular features. Am J Surg Pathol 2011, 35:474-483

3. Zukerberg LR, Harris NL, Young RH: Carcinomas of the urinary bladder simulating malignant lymphoma: a report of five cases. Am J Surg Pathol 1991, 15:569-576

4. Gulley ML, Amin MB, Nicholls JM, Banks PM, Ayala AG, Srigley JR, Eagan PA, Ro JY: Epstein-Barr virus is detected in undifferentiated nasopharyngeal carcinoma but not in lymphoepithelioma-like carcinoma of the urinary bladder. Hum Pathol 1995, 26:1207-1214

5. Yoshino T, Ohara S, Moriyama H: Lymphoepithelioma-like carcinoma of the urinary bladder: a case report and review of the literature. BMC Res Notes 2014, 7:779

6. Jessurun J, Romero-Guadarrama M, Manivel JC: Medullary adenocarcinoma of the colon: clinicopathologic study of 11 cases. Hum Pathol 1999, 30:843-848

7. Gafà R, Maestri I, Matteuzzi M, Santini A, Ferretti S, Cavazzini L, Lanza G: Sporadic colorectal adenocarcinomas with high-frequency microsatellite instability. Cancer 2000, 89:2025-2037

8. Lanza G, Gafà R, Matteuzzi M, Santini A: Medullary-type poorly differentiated adenocarcinoma of the large bowel: a distinct clinicopathologic entity characterized by microsatellite instability and improved survival. J Clin Oncol 1999, 17:2429

9. Rosenbaum MW, Bledsoe JR, Morales-Oyarvide V, Huynh TG, Mino-Kenudson M: PD-L1 expression in colorectal cancer is associated with microsatellite instability, BRAF mutation, medullary morphology and cytotoxic tumor-infiltrating lymphocytes. Mod Pathol 2016, 29:1104-1112

10. Inman BA, Sebo TJ, Frigola X, Dong H, Bergstralh EJ, Frank I, Fradet Y, Lacombe L, Kwon ED: PD-L1 (B7-H1) expression by urothelial carcinoma of the bladder and BCG-induced granulomata: associations with localized stage progression. Cancer 2007, 109: 1499-1505

11. Pichler R, Heidegger I, Fritz J, Danzl M, Sprung S, Zelger B, Brunner A, Pircher A: PD-L1 expression in bladder cancer and metastasis and its influence on oncologic outcome after cystectomy. Oncotarget 2017, 8:66849-66864

12. Sjödahl G, Lauss M, Lövgren K, Chebil G, Gudjonsson S, Veerla S, Patschan O, Aine M, Fernö M, Ringnér M, Månsson W, Liedberg F, Lindgren D, Höglund M: A molecular taxonomy for urothelial carcinoma. Clin Cancer Res 2012, 18:3377-3386

13. Choi W, Porten S, Kim S, Willis D, Plimack ER, Hoffman-Censits J, Roth B, Cheng T, Tran M, Lee I-L, Melquist J, Bondaruk J, Majewski T, Zhang S, Pretzsch S, Baggerly K, Siefker-Radtke A, Czerniak B, Dinney CPN, McConkey DJ: Identification of distinct basal and luminal subtypes of muscle-invasive bladder cancer with different sensitivities to frontline chemotherapy. Cancer Cell 2014, 25:152-165

14. Damrauer JS, Hoadley KA, Chism DD, Fan C, Tiganelli CJ, Wobker SE, Yeh JJ, Milowsky MI, Iyer G, Parker JS, Kim WY: Intrinsic subtypes of high-grade bladder cancer reflect the hallmarks of breast cancer biology. Proc Natl Acad Sci U S A 2014, 111: $3110-3115$

15. Robertson AG, Kim J, Al-Ahmadie H, Bellmunt J, Guo G, Cherniack AD, et al: Comprehensive molecular characterization of muscle-invasive bladder cancer. Cell 2017, 171:540-556.e25

16. Benhamou S, Allory Y, Sibony M, Krucker C, Laplanche A, Kamoun A, Biton A, Herault A, Ofualuka K, de Reynies A, Reyal F, Lepage M-L, Caillault A, Paoletti X, Molinie V, Neuzillet Y, 
Soyeux P, Elarouci N, Chapeaublanc E, Lebret T, Radvanyi F, Vordos D, Bernard-Pierrot I, Maille P, Letouze E, Southgate J, Rebouissou S, Denoux Y: EGFR as a potential therapeutic target for a subset of muscle-invasive bladder cancers presenting a basal-like phenotype. Sci Transl Med 2014, 6:244ra91

17. Lerner SP, McConkey DJ, Hoadley KA, Chan KS, Kim WY, Radvanyi F, Höglund M, Real FX: Bladder cancer molecular taxonomy: summary from a consensus meeting. Bladder Cancer 2016, 2: $37-47$

18. Seiler R, Ashab HAD, Erho N, van Rhijn BWG, Winters B, Douglas J, Van Kessel KE, Fransen van de Putte EE, Sommerlad M, Wang NQ, Choeurng V, Gibb EA, Palmer-Aronsten B, Lam LL, Buerki C, Davicioni E, Sjödahl G, Kardos J, Hoadley KA, Lerner SP, McConkey DJ, Choi W, Kim WY, Kiss B, Thalmann GN, Todenhöfer T, Crabb SJ, North S, Zwarthoff EC, Boormans JL, Wright J, Dall'Era M, van der Heijden MS, Black PC: Impact of molecular subtypes in muscle-invasive bladder cancer on predicting response and survival after neoadjuvant chemotherapy. Eur Urol 2017, 72:544-554

19. Ross JS, Wang K, Gay LM, Al-Rohil RN, Nazeer T, Sheehan CE, Jennings TA, Otto GA, Donahue A, He J, Palmer G, Ali S, Nahas M, Young G, LaBrecque E, Frampton G, Erlich R, Curran JA, Brennan K, Downing SR, Yelensky R, Lipson D, Hawryluk M, Miller VA, Stephens PJ: A high frequency of activating extracellular domain ERBB2 (HER2) mutation in micropapillary urothelial carcinoma AC. Clin Cancer Res 2014, 20:68-75

20. Guo CC, Dadhania V, Zhang L, Majewski T, Bondaruk J, Sykulski M, Wronowska W, Gambin A, Wang Y, Zhang S, FuentesMattei E, Kamat AM, Dinney C, Siefker-Radtke A, Choi W, Baggerly KA, McConkey D, Weinstein JN, Czerniak B: Gene expression profile of the clinically aggressive micropapillary variant of bladder cancer. Eur Urol 2016, 70:611-620

21. Al-Ahmadie HA, Iyer G, Lee BH, Scott SN, Mehra R, Bagrodia A, Jordan EJ, Gao SP, Ramirez R, Cha EK, Desai NB, Zabor EC, Ostrovnaya I, Gopalan A, Chen YB, Fine SW, Tickoo SK, Gandhi A, Hreiki J, Viale A, Arcila ME, Dalbagni G, Rosenberg JE, Bochner BH, Bajorin DF, Berger MF, Reuter VE, Taylor BS, Solit DB: Frequent somatic CDH1 loss-of-function mutations in plasmacytoid variant bladder cancer. Nat Genet 2016, 48:356-358

22. Sjödahl G, Eriksson P, Liedberg F, Höglund M: Molecular classification of urothelial carcinoma: global mRNA classification versus tumour-cell phenotype classification. J Pathol 2017, 242: $113-125$

23. Kulangara K, Zhang N, Corigliano E, Guerrero L, Waldroup S, Jaiswal D, Jansson M, Shah S, Hanks D, Wang J, Lunceford J, Savage MJ, Juco J, Emancipator K: Clinical utility of the combined positive score for programmed death ligand-1 expression and the approval of pembrolizumab for treatment of gastric cancer. Arch Pathol Lab Med 2019, 143:330-337

24. R Core Team: R: A Language and Environment for Statistical Computing. Vienna Austria, R Foundation for Statistical Computing, 2019

25. Frankish A, Diekhans M, Ferreira AM, Johnson R, Jungreis I, Loveland J, et al: GENCODE reference annotation for the human and mouse genomes. Nucleic Acids Res 2019, 47:D766-D773

26. Dobin A, Davis CA, Schlesinger F, Drenkow J, Zaleski C, Jha S, Batut P, Chaisson M, Gingeras TR: STAR: ultrafast universal RNAseq aligner. Bioinformatics 2013, 29:15-21

27. Patro R, Duggal G, Love MI, Irizarry RA, Kingsford C: Salmon provides fast and bias-aware quantification of transcript expression. Nat Methods 2017, 14:417-419

28. Soneson C, Love MI, Robinson MD: Differential analyses for RNAseq: transcript-level estimates improve gene-level inferences. F1000Res 2016, 4:1521

29. Durinck S, Spellman PT, Birney E, Huber W: Mapping identifiers for the integration of genomic datasets with the R/Bioconductor package biomaRt. Nat Protoc 2011, 4:1184-1191
30. Love MI, Huber W, Anders S: Moderated estimation of fold change and dispersion for RNA-seq data with DESeq2. Genome Biol 2014, 15:1-21

31. Leek JT, Johnson WE, Parker HS, Jaffe AE, Storey JD: The SVA package for removing batch effects and other unwanted variation in high-throughput experiments. Bioinformatics 2012, 28:882-883

32. Gu Z, Eils R, Schlesner M: Complex heatmaps reveal patterns and correlations in multidimensional genomic data. Bioinformatics 2016, 32:2847-2849

33. Wickham H: ggplot2: Elegant Graphics for Data Analysis. New York, NY, Springer-Verlag, 2016

34. Kardos J, Chai S, Mose LE, Selitsky SR, Krishnan B, Saito R, Iglesia MD, Milowsky MI, Parker JS, Kim WY, Vincent BG: Claudin-low bladder tumors are immune infiltrated and actively immune suppressed. JCI Insight 2016, 1:1-17

35. Tibshirani R, Hastie T, Narasimhan B, Chu G: Diagnosis of multiple cancer types by shrunken centroids of gene expression. Proc Natl Acad Sci U S A 2002, 99:6567-6572

36. Iglesia MD, Vincent BG, Parker JS, Hoadley KA, Carey LA, Perou CM, Serody JS: Prognostic B-cell signatures using mRNA-seq in patients with subtype-specific breast and ovarian cancer. Clin Cancer Res 2014, 20:3818-3829

37. Subramanian A, Subramanian A, Tamayo P, Tamayo P, Mootha VK, Mootha VK, Mukherjee S, Mukherjee S, Ebert BL, Ebert BL, Gillette Ma, Gillette Ma, Paulovich A, Paulovich A, Pomeroy SL, Pomeroy SL, Golub TR, Golub TR, Lander ES, Lander ES, Mesirov JP, Mesirov JP: Gene set enrichment analysis: a knowledgebased approach for interpreting genome-wide expression profiles. Proc Natl Acad Sci U S A 2005, 102:15545-15550

38. Liberzon A, Birger C, Thorvaldsdóttir H, Ghandi M, Mesirov JP, Tamayo P: The molecular signatures database hallmark gene set collection. Cell Syst 2015, 1:417-425

39. Selitsky SR, Mose LE, Marron D, Parker JS, Dittmer DP: EpsteinBarr virus-positive cancers show altered B-cell clonality. mSystems 2018, 3:1-10

40. Klijn C, Durinck S, Stawiski EW, Haverty PM, Jiang Z, Liu H, Degenhardt J, Mayba O, Gnad F, Liu J, Pau G, Reeder J, Cao Y, Mukhyala K, Selvaraj SK, Yu M, Zynda GJ, Brauer MJ, Wu TD, Gentleman RC, Manning G, Yauch RL, Bourgon R, Stokoe D, Modrusan Z, Neve RM, De Sauvage FJ, Settleman J, Seshagiri S, Zhang Z: A comprehensive transcriptional portrait of human cancer cell lines. Nat Biotechnol 2015, 33:306-312

41. Prat A, Karginova O, Parker JS, Fan C, He X, Bixby L, Harrell JC, Roman E, Adamo B, Troester M, Perou CM: Characterization of cell lines derived from breast cancers and normal mammary tissues for the study of the intrinsic molecular subtypes. Breast Cancer Res Treat 2013, 142:237-255

42. Bindea G, Mlecnik B, Tosolini M, Kirilovsky A, Waldner M, Obenauf AC, Angell H, Fredriksen T, Lafontaine L, Berger A, Bruneval $\mathrm{P}$, Fridman WH, Becker C, Pagès F, Speicher MR, Trajanoski Z, JÔ Galon: Spatiotemporal dynamics of intratumoral immune cells reveal the immune landscape in human cancer. Immunity 2013, 39:782-795

43. Kardos J, Wobker SE, Woods ME, Nielsen ME, Smith AB, Wallen EM, Pruthi RS, Hayward MC, McGinty KA, GrilleyOlson JE, Patel NM, Weck KE, Black P, Parker JS, Milowsky MI, Hayes DN, Kim WY: Comprehensive molecular characterization of urachal adenocarcinoma reveals commonalities with colorectal cancer, including a hypermutable phenotype. JCO Precis Oncol 2017, 1: $1-12$

44. Wasco MJ, Daignault S, Zhang Y, Kunju LP, Kinnaman M, Braun T, Lee CT, Shah RB: Urothelial carcinoma with divergent histologic differentiation (mixed histologic features) predicts the presence of locally advanced bladder cancer when detected at transurethral resection. Urology 2007, 70:69-74

45. Cai T, Tiscione D, Verze P, Pomara G, Racioppi M, Nesi G, Barbareschi M, Brausi M, Gacci M, Luciani LG, Liguori G, 
Gontero P, Campodonico F, Simonato A, Boddi V, Di Stasi SM, Colombo R, Serretta V, Carmignani G, Malossini G, Altieri V, Carini M, Terrone C, Bassi P, Montorsi F, Ficarra V, Selli C, Mirone V, Bartoletti R: Concordance and clinical significance of uncommon variants of bladder urothelial carcinoma in transurethral resection and radical cystectomy specimens. Urology 2014, 84: $1141-1146$

46. Moch H, Cubilla AL, Humphrey PA, Reuter VE, Ulbright TM: The 2016 WHO classification of tumours of the urinary system and male genital organs-part A: renal, penile, and testicular tumours. Eur Urol 2016, 70:93-105

47. Amin MB: Histological variants of urothelial carcinoma: diagnostic, therapeutic and prognostic implications. Mod Pathol 2009, 22 Suppl 2:S96-S118

48. Rosenberg JE, Hoffman-Censits J, Powles T, Van Der Heijden MS, Balar AV, Necchi A, Dawson N, O'Donnell PH, Balmanoukian A, Loriot Y, Srinivas S, Retz MM, Grivas P, Joseph RW, Galsky MD, Fleming MT, Petrylak DP, Perez-Gracia JL, Burris HA, Castellano D, Canil C, Bellmunt J, Bajorin D, Nickles D, Bourgon R, Frampton GM, Cui N, Mariathasan S, Abidoye O, Fine GD, Dreicer R: Atezolizumab in patients with locally advanced and metastatic urothelial carcinoma who have progressed following treatment with platinum-based chemotherapy: a single-arm, multicentre, phase 2 trial. Lancet 2016, 387:1909-1920

49. Sharma P, Retz M, Siefker-Radtke A, Baron A, Necchi A, Bedke J, Plimack ER, Vaena D, Grimm MO, Bracarda S, Arranz JÁ, Pal S, Ohyama C, Saci A, Qu X, Lambert A, Krishnan S, Azrilevich A, Galsky MD: Nivolumab in metastatic urothelial carcinoma after platinum therapy (CheckMate 275): a multicentre, single-arm, phase 2 trial. Lancet Oncol 2017, 18:312-322

50. Bellmunt J, de Wit R, Vaughn DJ, Fradet Y, Lee J-L, Fong L, Vogelzang NJ, Climent MA, Petrylak DP, Choueiri TK, Necchi A, Gerritsen W, Gurney H, Quinn DI, Culine S, Sternberg CN, Mai Y, Poehlein CH, Perini RF, Bajorin DF: Pembrolizumab as second-line therapy for advanced urothelial carcinoma. N Engl J Med 2017, 376: $1015-1026$

51. Massari F, Di Nunno V, Cubelli M, Santoni M, Fiorentino M, Montironi R, Cheng L, Lopez-Beltran A, Battelli N, Ardizzoni A: Immune checkpoint inhibitors for metastatic bladder cancer. Cancer Treat Rev 2018, 64:11-20

52. Choi W, Ochoa A, McConkey DJ, Aine M, Höglund M, Kim WY, Real FX, Kiltie AE, Milsom I, Dyrskjøt L, Lerner SP: Genetic alterations in the molecular subtypes of bladder cancer: illustration in the Cancer Genome Atlas dataset. Eur Urol 2017, 72:354-365 